\title{
Parasitic myositis in tropical Australia
}

\author{
Ron Basuroy, Robert Pennisi, Thomas Robertson, Robert Norton, John Stokes, Jon Reimers and John Archer
}

\begin{abstract}
Three patients with Australian parasitic myositis caused by the muspiceoid nematode Haycocknema perplexum are described. Treatment with albendazole led to a slow and incomplete recovery, but treatment with steroids caused life-threatening deterioration. (MJA 2008: 188: 254-256)
\end{abstract}

\section{Clinical records}

\section{Patient 1}

A 23-year-old woman presented to Cairns Base Hospital with a history of 2 years of insidiously progressive weakness, including 1 year of difficulty swallowing. She weighed $42 \mathrm{~kg}$, having lost $18 \mathrm{~kg}$ in recent years. There had been no myalgia.

The patient had grown up in Queensland and was living in Innisfail, but had travelled around Australia in the 3 previous years, visiting Western Australia, New South Wales and Victoria.

Although not acutely unwell or febrile, the patient was thin, with limb weakness and proximal and distal wasting. The facial muscles were wasted and weak, but palatal and ocular movements were normal. She was unable to stand from sitting without using her arms and had bilateral foot drop, but toe extension was preserved (Box 1). Reflexes were absent at the ankles and depressed elsewhere. Sensation appeared intact.

Haematological testing showed eosinophilia $\left(1.1 \times 10^{9} / \mathrm{L}\right.$; reference range $\left.[\mathrm{RR}],<0.4 \times 10^{9} / \mathrm{L}\right)$, which was found to have been known since 1998. It had been assumed to be due to intestinal parasites, a common problem in North Qld, but results of subsequent serology testing for Strongyloides were negative, as were stool samples.

The serum creatine kinase (CK) level was high (1370 U/L; RR, <160 U/L), and there was mild derangement of liver transaminases (alanine aminotransferase, 60 U/L; RR, <40 U/L); aspartate aminotransferase, $52 \mathrm{U} / \mathrm{L} ; \mathrm{RR}$ $<35 \mathrm{U} / \mathrm{L})$. Levels of inflammatory markers were also high (erythrocyte sedimentation rate, $50 \mathrm{~mm} / \mathrm{h}$; RR 0-20 mm/h; C-reactive protein, $37 \mathrm{mg} / \mathrm{L} ; \mathrm{RR},<5 \mathrm{mg} / \mathrm{L})$. Electromyography showed low-amplitude polyphasic potentials, consistent with myopathy, and nerve conduction studies showed a mild coexistent sensory neuropathy.

A biopsy of the quadriceps showed the muscle to be pale, flaccid and atrophic. On examination of paraffin sections, the muscle appeared highly abnormal; several muscle fibres contained sarcoplasmic nematode parasites characterised by a simple cephalic end and a sharply tapered caudal end. Unlike other nematodes that cause myositis, these parasites were not encysted. There was patchy interstitial and perivascular inflammation, pre- dominantly with lymphocytes, and numerous necrotic fibres showing florid myophagia (Box 2). The parasite stained red with Gomori's trichrome and positive with ATPase and cytochrome oxidase. Electron microscopy showed scattered sarcoplasmic parasites. Gravid female parasites were characterised by a thin cuticle, a central triradiated oesophageal lumen, and ova (Box 3). The parasite was identified as the nematode Haycocknema perplexum. ${ }^{1}$

The patient was treated with albendazole $400 \mathrm{mg}$ twice daily for 8 weeks. Percutaneous endoscopic gastrostomy provided nutritional support initially. Steroids were not used at any stage of treatment. After 2 weeks, CK had fallen to $235 \mathrm{U} / \mathrm{L}$ and C-reactive protein to $1.1 \mathrm{mg} / \mathrm{L}$. After 2 months, the patient remained diffusely weak, with ongoing dysphagia, but was able to stand from sitting.

\section{A 23-year-old woman with progressive muscle weakness (Patient 1)}

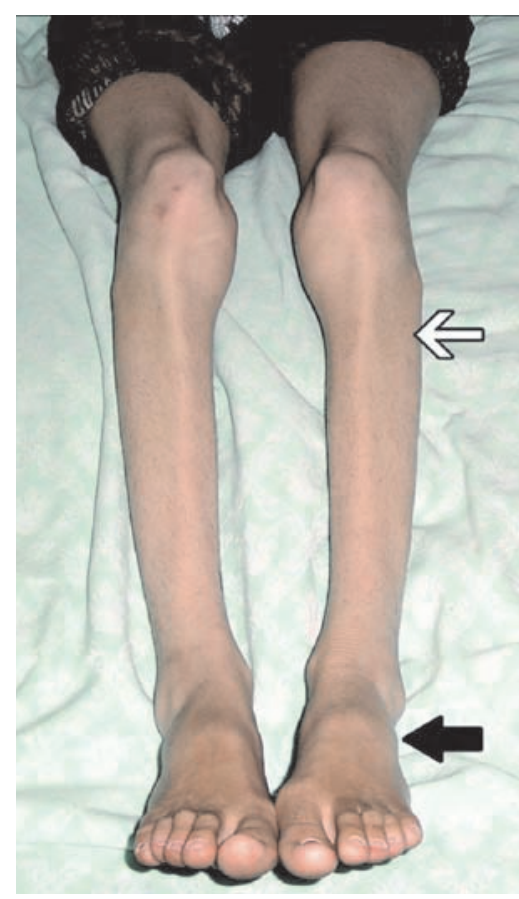

The patient had diffuse wasting of major lower limb muscle groups, including the quadriceps. The white arrow shows wasting of the tibialis anterior muscle, which caused foot drop. The black arrow shows the well preserved digitorum brevis muscle, accounting for preserved toe extension.
2 Micrographs of skeletal muscle (Patient 1)
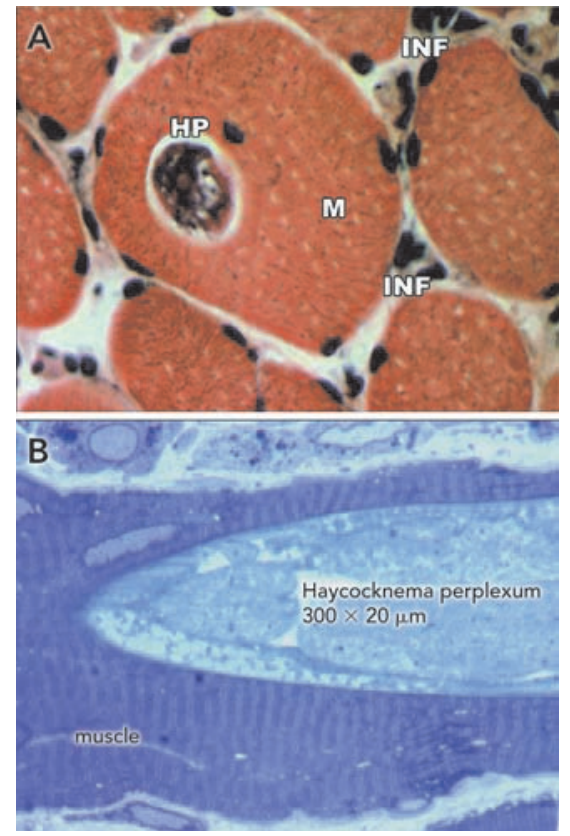

A: Transverse section of quadriceps muscle fibres (M) shows a nematode, Haycocknema perplexum (HP), in cytoplasm, surrounded by interstitial chronic inflammatory cells (INF) (haematoxylin and eosin stain; original magnification, $\times 400$ ).

$B$ : Longitudinal section shows parasitic nematode lying within the muscle fibre (toluidine blue stain; original magnification, $x$ 1000) 


\section{Electron micrographs of Haycocknema perplexum nematode}
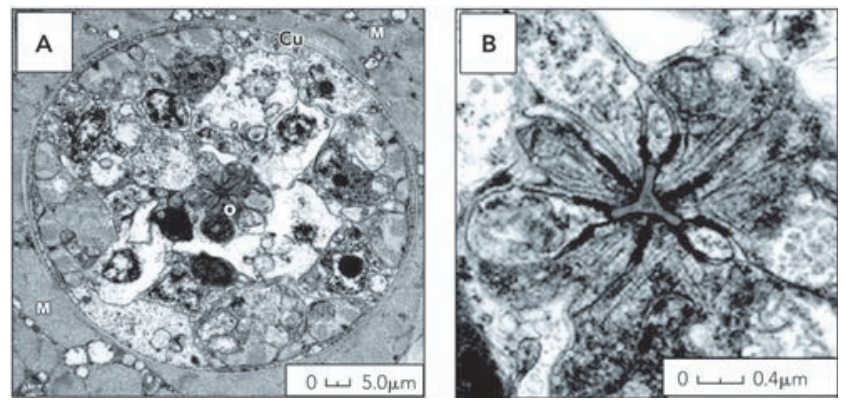

A: Cross-section through nematode in skeletal muscle (M), showing cuticle (Cu) and oesophagus (O) (original magnification, $x 2500$ ).

B: Enlargement shows cuticularised triradiated lumen of the oesophagus (original magnification, $\times 20000$ ).

Two months after she had completed treatment, the patient's CK began to rise, without eosinophilia. A second biopsy, of the deltoid muscle, did not show any live parasites, but showed numerous lysosomal structures, consistent with resorption of dead parasites. Significant replacement of muscle with collagenous connective tissue was noted. Twelve months after treatment, the patient had significant ongoing muscle weakness and persistently high CK levels (about $300 \mathrm{U} / \mathrm{L}$ ), but normal eosinophil counts.

\section{Patient 2}

A 61-year-old man was admitted to a Townsville hospital for investigation of a 3-year history of slowly progressive dysphagia and dysarthria, and 1 year of limb weakness. Concurrent medical problems included diabetes mellitus, renal impairment and biliary duct ectasia. Six months before presentation, hypercalcaemia had been noted and briefly treated with oral prednisone for presumed sarcoidosis, although serum angiotensin-converting enzyme levels were normal.

The patient had grown up in Tasmania, but had moved to Mackay in North Qld more than 20 years earlier, and had not travelled outside the region since.

On examination, he was unwell and cachectic, with bilateral facial and palatal weakness, diffuse limb weakness and wasting and depressed reflexes, but normal sensation. On the basis of elevated CK (1263 U/L) and inflammatory markers, high-dose oral prednisone was commenced for presumed polymyositis, pending muscle biopsy results. In retrospect, peripheral eosinophilia was known to have been present for at least 2 years. After the patient had received steroids, the eosinophil count normalised. Within days, he deteriorated, requiring admission to intensive care for ventilatory support.

After the muscle biopsy results showed parasitic myositis, steroids were gradually withdrawn and albendazole was commenced. The muscle function stabilised, but a biopsy 4 weeks later showed live $H$. perplexum nematodes. Further biopsy after 9 weeks of treatment showed no live nematodes.

The intensive care stay was complicated by dependence on ventilation, sepsis, pneumonia, renal and hepatic failure and encephalopathy. Seven months later he was discharged to a regional hospital for convalescence but died from complications of sepsis and renal failure.

\section{Patient 3}

A 61-year-old man from Mackay, Qld, was admitted to Townsville Hospital with a 2-year history of hand cramping, progressive diffuse weakness eventually causing difficulty climbing stairs, and 1 year of dysphagia.

He had lived in North Qld all his life, and had never travelled to Victoria or Tasmania. He had never consumed the meat of native animals or other "bush tucker". He was diffusely thin and weak, with bilateral incomplete foot drop and depressed reflexes, but intact facial strength and normal ocular movements.

The serum CK level was $1230 \mathrm{U} / \mathrm{L}$ and, in retrospect, had been 1000 U/L 2 years earlier, but had not been investigated. Alanine aminotransferase was $69 \mathrm{U} / \mathrm{L}(\mathrm{RR},<45 \mathrm{U} / \mathrm{L})$; aspartate aminotransferase, $67 \mathrm{U} / \mathrm{L}(\mathrm{RR}<40 \mathrm{U} / \mathrm{L})$; and peripheral blood eosinophils, $1.36 \times 10^{9} / \mathrm{L}\left(\mathrm{RR},<0.4 \times 10^{9} / \mathrm{L}\right)$. Review of earlier investigations confirmed persistent eosinophilia over the prior 42 months, at least.

Muscle biopsy confirmed H. perplexum. He received 8 weeks' oral treatment with albendazole. Steroids were not given. Four months later there was some improvement in limb strength and swallowing, although his CK level remained high.

\section{Discussion}

Although $H$. perplexum myopathy has previously been reported, in two people from Tasmania, 1,2 the patients we report had spent

4 Clinical and laboratory features of myopathy caused by Haycocknema perplexum

\begin{tabular}{|c|c|c|c|c|c|c|c|}
\hline Patient & $\begin{array}{l}\text { Place and year } \\
\text { of diagnosis }\end{array}$ & Prior travel & $\begin{array}{c}\text { Duration of } \\
\text { symptoms (years) }\end{array}$ & Dysphagia & CK level $(U / L)^{\star}$ & $\begin{array}{l}\text { Eosinophil count } \\
\qquad\left(\times 10^{9} / \mathrm{L}\right)^{\dagger}\end{array}$ & Outcome \\
\hline 1 & North Qld, 2005 & Extensive & 2 & ++ & 1370 & 1.1 & Weak, CK $300 \mathrm{U} / \mathrm{L}$ \\
\hline 2 & North Qld, 2004 & None in 20 years & 3 & ++ & 1263 & High & Weak, died \\
\hline 3 & North Qld, 2006 & Nil & 2 & + & 1230 & 1.36 & Weak, CK $\sim 250 \mathrm{U} / \mathrm{L}$ \\
\hline \multicolumn{8}{|c|}{ Previously reported cases ${ }^{2}$} \\
\hline A & Tasmania, 1996 & $\begin{array}{l}\text { Extensive, including } \\
\text { Far North Qld }\end{array}$ & 1.5 & ++ & 1586 & 2.0 & Weak, CK $280 \mathrm{U} / \mathrm{L}$ \\
\hline B & Tasmania, 1994 & $\begin{array}{c}\text { Extensive, including } \\
\text { northern Australia }\end{array}$ & 5 & + & 2168 & 0.8 & Good recovery \\
\hline
\end{tabular}

$\mathrm{CK}=$ creatine kinase. $\mathrm{Qld}=$ Queensland.$+=$ mild-moderate. $++=$ moderate-severe. ${ }^{\star}$ Reference range $(\mathrm{RR}),<160 \mathrm{U} / \mathrm{L} . \dagger \mathrm{RR},<0.4 \times 10^{9} / \mathrm{L}$. 
most or all of the past few years in tropical northern Australia. We contacted the physicans caring for the earlier patients, who confirmed that they, too, had been exposed to tropical Australia (Box 4). One had travelled extensively in Cape York, Far North Qld, and Kakadu National Park, Northern Territory, 5 years before diagnosis. Around that time, he developed a non-specific illness with raised liver transaminase levels, and liver biopsy showed mild reactive hepatitis with eosinophils. The other was a botanist who had visited the Northern Territory, Tasmania, Europe, Kenya and Indonesia on fieldwork before developing muscle weakness.

Clinical features common to $H$. perplexum myositis patients were the insidious onset of diffuse limb weakness and dysphagia. The documentation of persistent eosinophilia up to 7 years before diagnosis suggests that infection may be subclinical for years. Consistent clinical signs have been diffuse limb wasting and weakness, including bilateral foot drop. Uniform abnormalities included an elevated serum CK, blood eosinophilia and mild elevations of liver transaminases. Although muscle biopsy is required to confirm diagnosis, in one reported case the initial biopsy did not reveal parasites, but a second biopsy after steroid treatment showed $H$. perplexum.

H. perplexum is a minute nematode measuring about $350 \mu \mathrm{m}$ by $20 \mu \mathrm{m}$. The parasite appears to be able to complete its life cycle within human muscle, as adult nematodes are found within the sarcoplasm of muscle cells, while larvae are found both inside and outside myofibres. ${ }^{3}$ Electron microscopy shows the characteristic features of $H$. perplexum: a cuticularised triradiated oesophagusintestine terminating in dark refringent granules (trophosomes). ${ }^{1}$ The outer cuticle is thin and has exterior corrugations. The male is shorter and narrower than the female, with the testes occupying $50 \%-60 \%$ of its body. The gravid female has paired uteri where 12 to 24 ova develop into larvae. Auto-reinfection is thought to occur when third-stage larvae escape by bursting through the female nematode's body, a phenomenon known as endotokia matricida. The resulting damaged myofibres incite an intense inflammatory reaction resulting in myophagia.

H. perplexum was first described in the late 1990s, having been identified as the cause of a case of human myositis. ${ }^{2}$ The parasite is a member of the Robertdollfusidae family of Muspiceoidea nematodes. Distinct species of Muspiceoidea nematodes have been found in the tissues of various Australian vertebrates, ${ }^{4}$ including mice, ${ }^{3}$ bats, ${ }^{5}$ kangaroos and wallabies, ${ }^{6}$ and koalas. ${ }^{7}$ In animals, there is some evidence for cutaneous penetration as the mechanism of infection. ${ }^{3}$

The mechanism by which humans acquire $H$. perplexum is unknown. Other parasitic myopathies such as trichinosis and cysticercosis are acquired through consumption of poorly cooked meats. Intestinal parasites, including Strongyloides, may be acquired via direct cutaneous penetration, such as when walking barefoot, a common practice in tropical Australia. Mosquitoes, which are very common in tropical Australia during the wet season, could also be a vector for infection.

Treatment with albendazole improved muscle strength in all three cases of $H$. perplexum myopathy, although recovery was slow and incomplete. A biopsy after 4 weeks of treatment showed live nematodes. After 9 weeks of treatment, no live nematodes were seen on biopsy, correlating with a reduction in CK levels and peripheral eosinophilia. Therefore, treatment for at least 8 weeks is suggested to prevent auto-reinfection. Treatment solely with albendazole, without concomitant steroid use, was not associated with adverse reactions from nematode death. Furthermore, steroid therapy resulted in deteriorating muscle function and delayed diagnosis by falsely normalising blood eosinophilia.

\section{Acknowledgement}

We thank Dr Philip Marshall for clinical care of Patient 1 and for assistance with manuscript preparation.

\section{Competing interests}

None identified.

\section{Author details}

Ron Basuroy, MRCP, Medical Registrar ${ }^{1}$

Robert Pennisi, FRACGP, Registrar ${ }^{2}$

Thomas Robertson, FRCPA, Pathologist ${ }^{2}$

Robert Norton, FRCPA, MRCP, Infectious Diseases Physician ${ }^{3}$

John Stokes, FJFICM, Intensivist, ${ }^{4}$ and Associate Professor ${ }^{5}$

Jon Reimers, FRACP, Neurologist ${ }^{3}$

John Archer, FRACP, PhD, Senior Lecturer, Medicine, 5,6 and

Neurologist ${ }^{1}$

1 Department of Medicine, Cairns Base Hospital, Cairns, QLD.

2 Department of Anatomical Pathology, Royal Brisbane Hospital,

Brisbane, QLD.

3 Department of Medicine, Townsville Hospital, Townsville, QLD.

4 Department of Intensive Care, Mater Hospital, Townsville, QLD.

5 Department of Medicine, James Cook University, Cairns and

Townsville, QLD.

6 Department of Medicine, University of Melbourne, Melbourne, VIC. Correspondence: jarcher@unimelb.edu.au

\section{References}

1 Spratt DM, Beveridge I, Andrews JRH, Dennett X. Haycocknema perplexum n. g., n. sp. (Nematoda: Robertdollfusidae): an intramyofibre parasite in man. Syst Parasitol 1999; 43: 123-131.

2 Dennett X, Siejka SJ, Andrews JR, et al. Polymyositis caused by a new genus of nematode. Med J Aust 1998; 168: 226-227.

3 Spratt DM, Haycock P, Boyden JM, Nicholas WL. Aspects of the life history of Muspicea borreli (Nematoda: Muspiceidae), parasite of the house mouse (Mus domesticus) in Australia. Parasite 2002; 9: 199-205.

4 Spratt DM. Australian ecosystems, capricious food chains and parasitic consequences for people. Int J Parasitol 2005; 35: 717-724.

5 Bain O, Chabaud AG. Sur les Muspiceidae (Nématoda-Doryliamina) [French]. Ann Parasitol Hum Comp 1979; 54: 207-225.

6 Spratt DM, Speare R. Durikainema macropi gen. et sp. nov. (Muspiceoidea: Robertdollfusidae). A remarkable nematode from Macropodidae (Marsupialia). Ann Parasitol Hum Comp 1982; 57: 53-62.

7 Spratt DM, Gill PA. Durikainema phascolarctin. sp. (Nematoda: Muspiceoidea: Robertdollfusidae) from the pulmonary arteries of the koala Phascolarctos cinereus with associated pathological changes. Syst Parasitol 1998; 39: 101-106.

(Received 23 Aug 2007, accepted 9 Dec 2007) 